\title{
Development and Operational Planning of Power Systems by Comparing Scenarios during Multi-Objective Optimization
}

\author{
P. Stefanov*, A. Savić And G. Dobrić \\ School of Electrical Engineering, University of Belgrade, Belgrade, Serbia
}

\begin{abstract}
Multi-objective optimization in development and operational planning of power systems becomes crucially important for system designers or operators. In addition, appropriate procedures increasingly involve comparison of scenarios, each with variables that have to be neither dimensionally nor qualitatively comparable. Selecting the best solution is usually performed posteriorly by comparing the results of different scenarios. In order to eliminate unnecessary calculations during the optimization, especially in the case of large number of scenarios, it is useful to eliminate the scenarios with bad indicators. This process can significantly reduce the computational time. It results in a timely elimination of all inferior scenarios, thus simplifying the decision-making. This paper analyses the methods and the efficiency of comparison of different scenarios being simultaneously processed using NSGA-II and MOPSO optimization procedures. The proposed procedures are discussed in the example of selecting the type, the size and the location of FACTS in transmission networks. Different scenarios consider the common types of these devices, wherein the number of devices depends on the system. The paper proposes the reduction of the optimization procedures. Having no significant influence on the final set of optimal solutions, the proposed modifications are significant and applicable.
\end{abstract}

DOI: 10.12693/APhysPolA.128.B-138

PACS: 88.80.H-

\section{Introduction}

Development and operational planning of power systems becomes practically impossible without the use of optimization methods. In order to solve such problems, large number of different mathematical programming techniques have been developed. List of the applied techniques for optimal power flow (OPF) only, becomes exceptionally long $[1,2]$. The need for the application of multi-criteria optimization has confirmed the importance of heuristic methods [3], since they provide efficient insight into trade-offs between different objectives [4]. While multi-objective optimization enables increasing number of criteria to be considered, the various possible ways of development and operation of power systems lead to the necessity of solving optimization problems by comparing a large number of scenarios. A simple example of the development of transmission network by building new transmission capacity, upgrade old ones or introduce additional control equipment [5] shows that the control variables in the optimization procedures of individual development scenarios need not to be either dimensionally or qualitatively comparable.

Comparison of scenarios in multi criteria optimization can be done by posterior comparison of different scenarios, according to the selected set of objective functions. In order to do so, the unique fitness function has to be defined for each scenario and considered in the optimization procedure of each one. Thus, scenario comparison provides a set of mutually comparable optimal solutions, which present trade-off between different objectives for

*corresponding author; e-mail: stefanov@etf.rs different scenarios. The only way to ensure the reliability of the results of the optimization procedure is to require consideration of increased number of scenarios, with a high probability of the occurrence of excessive scenarios. The solutions of these scenarios are not superior on any optimization criterion for any criterion combination. Obviously, all the calculations performed for these scenarios are unnecessary and they additionally burden the optimization procedure. Excluding the scenarios with inferior indicators can lead to a significant reduction of the optimization procedure and the computational time and simplification of the final decision for the decision-maker.

The procedure proposed in this paper provides a scenario comparison during the optimization procedure. The algorithm enables timely identification of the "excessive" scenarios and eliminates the need for further calculations on them. In addition, this paper compares the methods and efficiency of comparisons during parallel processing of a number of different scenarios, when using some different optimization procedures, such as the nondominated sorting genetic algorithm (NSGA-II) and the multi-objective particle swarm optimization (MOPSO).

The procedures are verified on the problem of development of transmission network by installing flexible AC transmission system (FACTS) devices. These devices require versatile mathematical formulations with the inability to create a unique set of optimization. Comparison of the operation of these devices is enabled using the unique set of objective functions, which provide the same representation of Pareto front for all the scenarios considered.

\section{Scenario comparison}

If the optimization includes different alternatives, the associated vectors can have different variable types and 
sizes. Formation of the unique vector in the abovementioned problem involves mixed integer/binary nonlinear programming, which may lead to practically unsolvable formulation if realistic modelling is used, with large computational requirements. In such cases, the implementation of computations with the comparison of different scenarios, named parallel scenario algorithm (PSA), is better solution. PSA, shown in Fig. 1, enables the formation of the unique Pareto front for all the considered scenarios with the possibility to optimally reduce the calculation by timely excluding the scenarios that have inferior solutions.

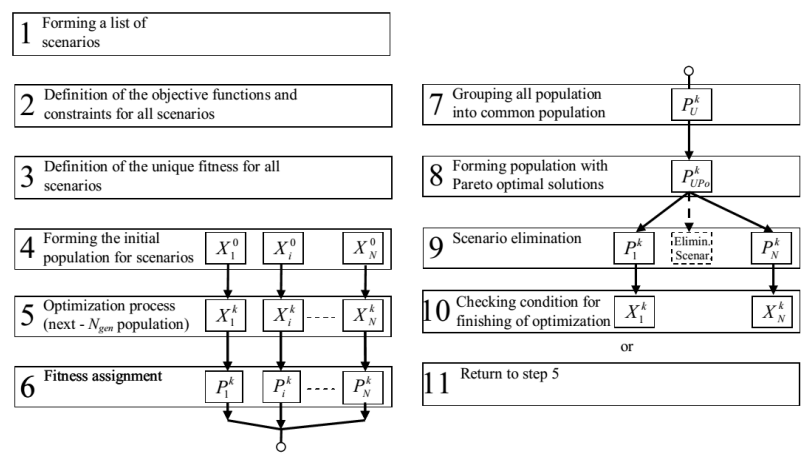

Fig. 1. PSA algorithm.

In the first step of the PSA algorithm, the optimization scenario list is created. For each considered scenario, in the second step, objective functions, constraints of inequality type and constraints of equality type are defined according to the formulation of the problem, while in the third step of PSA algorithm the unique fitness for all scenarios is defined. The possibility of comparison of scenarios with different control variables is provided with the adoption of a unique set of objective functions (control variables and all constraints may remain different) for all considered scenarios. This allows the determination of a single fitness function for all scenarios. Different scenarios can be compared at any stage of the optimization procedure using equivalent fitness and Pareto-based algorithms $[3,6]$.

The fourth and the fifth step, according to Fig. 1, are conducted independently for each considered scenario. While the fourth step presents the initialization of the optimization procedure for all scenarios defined in step one, the fifth step is conducted only for the scenarios that are selected for further consideration. In this step, the population created in $k$-th iteration for scenario $i$ is the result of the optimization procedure after the given number of generations $N_{\text {gen }}$, which defines the frequency of scenario comparison. The minimum value of this number is one, which implies comparison in each iteration, while the maximum value is equal to the total number of generations and implies comparison only at the end of the optimization procedure.

In the sixth step, uniquely defined fitness values are assigned to all population elements for each considered scenario. The next step groups populations of all scenarios into one single population. In the eighth step, only Pareto optimal solutions are kept according to the Pareto-based assignment. These solutions present the solutions of the optimization problem in the on-going iteration of PSA algorithm considering all the scenarios. The scenarios, which do not have any solutions in the created population, are eliminated in the ninth step.

During the scenario comparison, it should be taken into account that the optimization procedures can have different degree of convergence for different scenarios. The PSA method enables the comparison of scenarios analysed with different optimization algorithms. In this case, it is necessary to have insight into the flow of each of the algorithms with the eventual comparison of different stages, which occur during optimization, and their impact on the convergence. In this paper, such an analysis is carried out for two optimization procedures: NSGA-II and MOPSO.

Genetic algorithm (GA) search technique is an adaptive process, which requires continuous tracking of the search dynamics [3]. The choice of initial values for the crossover and mutation rates, and their tuning can significantly influence the optimization performance. These parameters can be adapted either manually or automatically. On the other hand, MOPSO algorithm depends on control parameters and optimization stage [7]. MOPSO algorithm is characterized by the existence of stages of the optimization process, depending on the number of particles, which are associated with "stock" Pareto optimal solutions in different iterations. This number is not constant, as in GA. In the first stage, the number of particles increases and decreases randomly. Second stage presents saturation and rapid increase in the number of particles, while third stage, stagnation, begins when the number of particles reaches the maximum value. During this stage, the solutions are "stretched" and distributed more evenly.

It can be concluded that when comparing scenarios during the optimization procedure, one should consider the influence that convergence speed (optimization stages) can have on the solutions of each scenario. One simple way of its consideration is based on the observation of the relationship of certain scenario solutions for a number of iterations. Instead of being directly eliminated, these scenarios can be declared as "candidates for elimination" and eliminated only if confirmed not to have Pareto optimal solutions in a specified number of consecutive iterations. Another way, also presented in this paper, is based on the definition of the index, which characterize the volume of the Pareto optimality of optimal solutions in a given iteration compared to the solutions of certain scenarios. The Pareto optimality of solutions is determined by definition [3]: "solution $\boldsymbol{x}^{*} \in \boldsymbol{X}$ is Pareto optimal, if and only if there is no other solution $\boldsymbol{x} \in \boldsymbol{X}$, such as $\boldsymbol{F}(\boldsymbol{x}) \leq \boldsymbol{F}\left(\boldsymbol{x}^{*}\right)$ and $F_{i}(\boldsymbol{x})<F_{i}\left(\boldsymbol{x}^{*}\right)$ for at least one objective function, where $\boldsymbol{X}$ stands for feasible space of control variables, $\boldsymbol{x}$ stands for control variables vector (solution in the space of control variables) and $\boldsymbol{F}$ stands 
for objective function vector (solution in the space of objective function)". Using this, the index for scenario $j$ is determined by the equation

$$
\gamma_{j}=\min _{i \in S_{j}} \frac{\left\|F\left(x_{i}\right)\right\|}{\max _{k}\left\|F\left(x_{k}^{*}\right)\right\|} .
$$

In Eq. (1), $S_{j}$ stands for the set of all solutions that belong to scenario $j$, while index $k$ is used for searching all Pareto optimal solutions in the given iteration. In case that $\gamma_{j} \geq \Upsilon$, where $\Upsilon$ is a number $\geq 1$, the considered scenario is excluded from further calculations.

\section{Optimization of electric power system} development with installation of FACTS devices

The rapid development of power electronics technology provides the opportunities to develop new power system equipment for better utilization of existing systems. During the last decade, a number of control devices under the term FACTS have been proposed and implemented. FACTS devices can be effectively used for power flow control, loop-flow control, load sharing among parallel corridors, voltage regulation, enhancement of transient stability and mitigation of system oscillations [8]. A vast number of FACTS devices from different generations with different dimensions of vector of control variables which are associated with individual devices [9], make this problem a characteristic example of possible applications of the abovementioned procedure. The most important devices' variables that can be taken as control variables in standard OPF calculations are shown in Table I, depending on the device modelling and the control modes.

TABLE I

FACTS devices control variable [8].

\begin{tabular}{c|c|c}
\hline \hline Device & Potential control variable & $\begin{array}{c}\text { Dim. of } \\
\text { contr. var. } \\
\text { vect. }\end{array}$ \\
\hline FS & $\theta_{\mathrm{FS}}, P_{k l}$ & 1 \\
SVC & $\alpha_{\mathrm{SVC}}, X_{\mathrm{SVC}}, U_{\mathrm{SVC}}, Q_{\mathrm{SVC}}, I_{\mathrm{SVC}}$ & 1 \\
TCSC & $\alpha_{\mathrm{TCSC}}, X_{\mathrm{TCSC}}, P_{k l}, I_{k l}$ & 1 \\
STATCOM & $m_{\mathrm{DC}}, \alpha, E_{\mathrm{dS}}, E_{\mathrm{qS}}, U_{\mathrm{S}}, Q_{\mathrm{S}}, I_{\mathrm{S}}$ & 1 \\
SSSC & $m_{\mathrm{DC}}, \alpha, E_{\mathrm{dR}}, E_{\mathrm{qR}}, P_{k l}, I_{k l}$ & 1 \\
$\mathrm{UPFC}$ & $m_{\mathrm{DCS}}, \alpha_{\mathrm{S}}, m_{\mathrm{DCR}}, \alpha_{\mathrm{R}}, E_{\mathrm{dS}}, E_{\mathrm{qS}}, E_{\mathrm{dR}}$, & 3 \\
& $E_{\mathrm{qR}}, E_{\mathrm{R}}, \delta_{\mathrm{R}}, U_{\mathrm{S}}, Q_{\mathrm{S}}, I_{\mathrm{S}}, P_{k l}, \theta_{k l}, Q_{k l}$ & \\
IPFC & $m_{\mathrm{DC} 1}, \alpha_{1}, m_{\mathrm{DC} 2}, \alpha_{2}, E_{\mathrm{d} 1}, E_{\mathrm{q} 1}, E_{\mathrm{d} 2}$, & 3 \\
& $E_{\mathrm{q} 2}, E_{1}, \delta_{1}, P_{k l}, \theta_{k l}, Q_{k l}$ &
\end{tabular}

As can be seen in Table I, most of the devices, depending on their configuration and control strategy definition, can have different control modes with different set of control variables. The selection of the device type determines the influence on the system performance. In the case of the combination of devices, their size and location has to be the result of multi-criteria optimization.

The problem is observed from the system point of view and different development strategies provide guidelines to which devices or their combinations give the optimal solution for the system. The proposed three objective functions provide analysis of the system operation through economic indicators related to the system active power losses $\left(f_{1}\right)$, through optimal-var planning $\left(f_{2}\right)$ and considering the system reliability $\left(f_{3}\right)$ by providing the congestion resolving [5].

Scenarios that can be considered refer to inclusion of individual devices or their combinations in the given system. Maximum number of devices considered in each scenario is determined according to the size and configuration of the IEEE 57 bus test system that was used to conduct the calculations. If all types of devices from Table I were considered, in the case of maximum three devices per scenario the number of possible scenarios would be 119, while in the case of four devices this number would raise to 329 . This leads to a conclusion that the proposed procedure has great implication if the comparison of all solutions for real power systems is needed.

The review of the results is limited to four scenarios due to simplicity and obviousness. These scenarios comprise characteristic shunt devices (SVC), series devices (TCSC) and combined (UPFC) FACTS devices. Starting analysis focuses on comparison of optimization procedures, using NSGA with constant and adaptively controlled parameters. Figure 2 shows the solutions of 2 SVC scenario in the plane of objective functions $f_{1}$ and $f_{2}$ (Fig. 2a), as well as $f_{1}$ and $f_{3}$ (Fig. 2b), after 40 iterations. Shown deviations are mainly related to the spread of solutions, hence not significantly affecting the dominance, thus ensuring their practically the same treatment as compared scenarios.

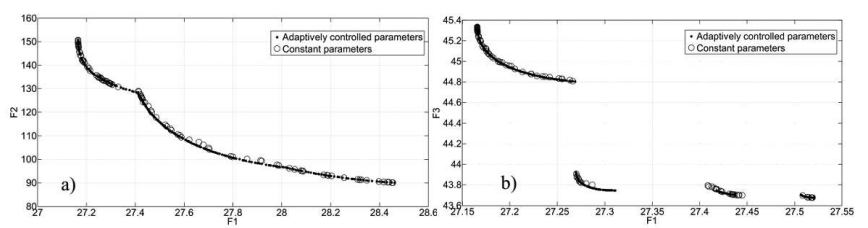

Fig. 2. Comparison of optimization procedures using NSGA for 2 SVC scenario with constant and adaptively controlled control parameters: (a) the plane of objective functions $f_{1}$ and $f_{2}$, (b) the plane of objective functions $f_{1}$ and $f_{3}$.

Figure 3 compares the results of NSGA and MOPSO for the same scenario. Due to the existence of different stages in the MOPSO optimization, this comparison is given through different stages in order to gain a better insight into the relative positions of the obtained solutions and the method of treating these solutions when comparing scenarios.

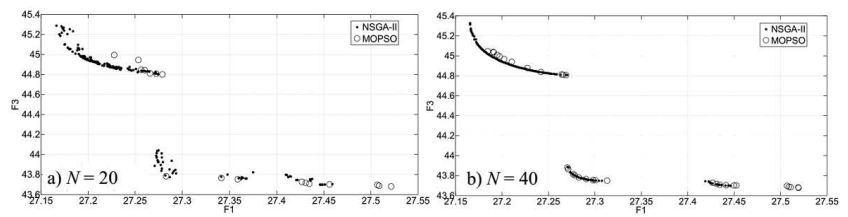

Fig. 3. Comparison of the results of NSGA and MOPSO for 2 SVC scenario in different stages of optimization (plane $f_{1}$ and $f_{3}$ ). 
The number of MOPSO optimal solutions varies, but despite these variations, as in the case of variation of control parameters, position of the solutions enables the same treatment for the Pareto optimality. Hence, both procedures can be used when comparing the solutions during the procedure.

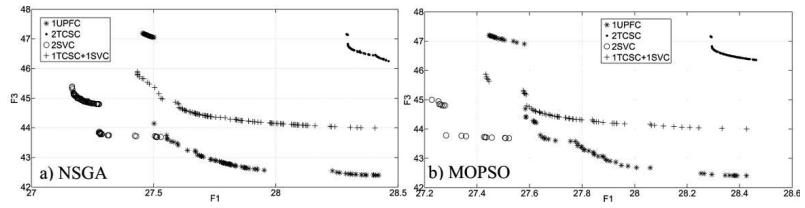

Fig. 4. The solutions of all four scenarios in the plane of objective functions $f_{1}$ and $f_{3}$ after 20 iterations.

Finally, Fig. 4a shows the solutions of all four scenarios in the plane of objective functions $f_{1}$ and $f_{3}$ after 20 iterations of NSGA, while Fig. 4b demonstrates the equivalent comparison using MOPSO. By comparing the results, it can be concluded that despite the small and varying number of solutions that remain in MOPSO storage, they are with similar geometric position. Besides, the value of the Pareto optimality index (given in Table II) is about the same at any time during the optimization. Obviously, this conclusion enables each of the optimization algorithms to be arbitrarily selected in PSA procedure.

\section{TABLE II}

Pareto optimality index during PSA.

\begin{tabular}{c|c|c|c|c}
\hline \hline Iteration of PSA & 1 UPFC & 2 TCSC & 2 SVC & $\begin{array}{c}1 \text { TSCS } \\
+1 \text { SVC }\end{array}$ \\
\hline 1st (10 gen) & 0.9858 & 1.0363 & 0.9870 & 1.0017 \\
2nd (20 gen) & 0.9838 & 1.0338 & 0.9849 & 1.0011 \\
10th (100 gen) & 0.9841 & & 0.9851 &
\end{tabular}

Applying the PSA with delayed elimination, already after twenty iterations of NSGA and/or MOPSO, only 2 scenarios with 1 UPFC and 2 SVC remain. Similar conclusion is made by tracking the Pareto optimality index in Table II, while the value of marginal indicator is set to one. The final appearance of the Pareto front in the plane of objective functions $f_{1}$ and $f_{3}$ is shown in Fig. 5a, while Fig. $5 \mathrm{~b}$ shows the Pareto optimal front (as the final result) in the space of all three objective functions.

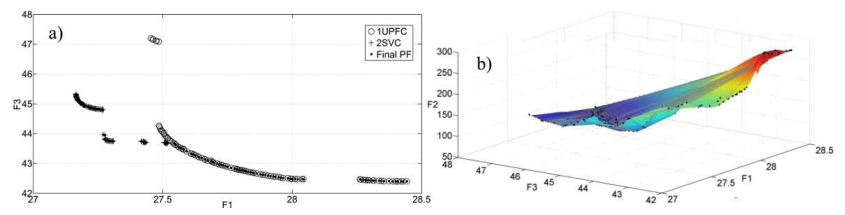

Fig. 5. The final Pareto front in the plane of objective functions $f_{1}$ and $f_{3}(\mathrm{a})$ and in the $3 \mathrm{D}(\mathrm{b})$.

\section{Conclusion}

The presented optimization procedure allows a comparison of solutions that include consideration of various strategies that cannot be modelled in the same way and which are connected to various control variables. It has enabled a comparative assessment of different strategies, their evaluation and definition of comparative advantage.

Implementation of PSA enables comparison of scenarios during the procedure and the elimination of scenarios with non-dominant solutions. The presented results confirm that this comparison can be done efficiently and with the application of different optimization algorithms such as NSGA-II and MOPSO, without affecting the selection of scenarios. The interrelation of solutions of different scenarios retains the same value throughout the optimization process, as indicated by the value of the proposed optimality index.

The application of this procedure to the presented problem indicates efficient selection of scenarios, thus clearly indicating good development strategy, in terms of all the aspects that are considered with the objective functions. In addition, a set of solutions that system planners leave as candidates for the final decision is significantly reduced, so that the final decision is significantly simplified and facilitated.

\section{References}

[1] J.A. Momoh, M.E. El-Hawary, R. Adapa, IEEE Trans. Power Syst. 14, 96 (1999).

[2] K.S. Pandya, S.K. Joshi, J. Theor. Appl. Inform. Technol. 4, 450 (2008).

[3] K. Deb, Multi-Objective Optimization Using Evolutionary Algorithms, Wiley, UK 2001.

[4] J.H. Chow, F.F. Wu, J.A. Momoh, Applied Mathematics for Restructured Electric Power Systems, Springer, USA 2005.

[5] J. Momoh, L. Mili, Economic Market Design and Planning for Electric Power Systems, IEEE Press, Wiley, New Jersey 2010.

[6] M.A. Abido, Electric Power Syst. Res. 79, 1105 (2009).

[7] M. Reyes-Sierra, C.A. Coello Coello, Int. J. Computat. Intellig. Res. 2, 287 (2006).

[8] E. Acha, C.R. Fuerte-Esquivel, H. Ambriz-Pérez, C. Angeles-Camacho, FACTS Modelling and Simulation in Power Networks, Wiley, Chichester 2004.

[9] G. Glanzmann, G. Andersson, in: Proc. 37 North American Power Symp., Ames (USA), Ames 2005, p. 141. 